\section{Anti-Ku syndrome with elevated CK: association with myocardial involvement in systemic sclerosis}

We read with great interest the paper by Spielmann et al describing a large cohort of anti-Ku-positive connective tissue disease patients. ${ }^{1}$ In their paper, the authors could identify two different subgroups of patients: 'anti-Ku with elevated CK patients', who are at risk of developing interstitial lung disease (ILD), and 'anti-Ku with anti-dsDNA patients', who are at risk of developing glomerulonephritis. Interestingly, the authors found an extremely low rate of myocarditis in both subgroups as only one patient in the C1 cluster ('elevated CK') was diagnosed with myocarditis. Of note, in our cohort of anti-Ku-positive systemic sclerosis (SSc) patients we found that the positivity for this rare antibody was invariably associated with myocardial inflammation. We indeed performed a retrospective review of anti-Ku-positive patients affected by SSc according to European League Against Rheumatism/American College of Rheumatology (EULAR/ACR) 2013 criteria $^{2}$ followed-up at two referral centres for SSc. We routinely performed anti-Ku in all SSc patients without any detected positivity for other SSc-related antibodies, that is, antitopoisomerase I, anticentromere and anti-RNA polymerase III. All SSc patients with suspected myocardial inflammation (new onset cardiac signs and/or symptoms, raised troponin $\mathrm{T}$ and/or $\mathrm{N}$-terminal pro-brain natriuretic peptide (NTproBNP)) routinely underwent cardiac magnetic resonance (CMR) and 24-hour-Holter-ECG tape. Myocarditis diagnosis was defined according to the Lake Louise Criteria ${ }^{3}$ on CMR. We identified four anti-Ku-positive SSc patients. All patients were female, and had been diagnosed with limited cutaneous SSc; mean age at diagnosis was $52.5 \pm 19.74$ years. In all cases, the anti-Ku positivity was confirmed by immunoblotting. Mean delay between myocarditis and SSc onset was 79.2 \pm 48.1 months. In three out of four patients (75\%), ILD was also present. Myositis was diagnosed in all patients by creatine kinase (CK) increase, electromyography and skeletal MRI, and in all cases it preceded the diagnosis of myocarditis. None of the patients had scleroderma renal crisis or pulmonary arterial hypertension. Two patients had subclinical presentation, while the other two had signs of heart failure. At presentation, troponin $\mathrm{T}$ serum levels were increased in all patients (mean levels: $82 \pm 42.11 \mathrm{ng} / \mathrm{L}$ ), and NTproBNP was slightly raised in three patients (mean levels $317.5 \pm 4.6 \mathrm{pg} / \mathrm{mL}$ ). At 24-hourHolter-ECG tape, three patients had also arrhythmic abnormalities, mainly mildly increased number of ventricular ectopic beats (VEBs) (mean 113 $\pm 56 / 24$ hours). CMR unequivocally showed late gadolinium enhancement abnormalities in all cases; Short TI Inversion Recovery (STIR) abnormalities with a non-ischaemic pattern, suggestive for myocardial oedema, were detectable in half of the patients. In two patients, moreover, pericardial effusion was also observed. Once myocarditis was diagnosed, three patients were started on mycophenolate mofetil (MMF) (2 g/day in all cases) and one patient was started on azathioprine $100 \mathrm{mg}$ /day, subsequently reduced to $50 \mathrm{mg} /$ day due to leucopoenia. Steroid pulses $(1 \mathrm{~g}$ methylprendisolone for 3 days) and then oral steroid therapy were also started in one patient with concomitant myositis relapse. After a median follow-up time of 12 months (range 8-24) in two patients was achieved an optimal disease control. Unfortunately though, in two patients MMF therapy was not able to curb myocardial inflammation. One patient had indeed to stop MMF therapy due to gastrointestinal complains and she could only tolerate low-dose MMF therapy $(500 \mathrm{mg} /$ day). After 3 months, she developed arrhythmic complications with frequent VEBs (2370/24hours) and she is being evaluated for implantable cardiac defibrillator (ICD) implantation. The other patient was also started or rituximab therapy due to poor myocardial, articular and muscular control with only partial benefit.

Our findings emphasised the reported association between skeletal myositis and myocarditis in SSc, ${ }^{45}$ and the positivity for anti-Ku antibodies seems to strengthen this important clinical relationship. Once confirmed in larger cohorts, this notion could be of great clinical value, since myositis in frequently detected in SSc patients and myocarditis is unequivocally associated with a dismal prognosis. ${ }^{45}$ Importantly though, myocarditis could be asymptomatic or clinically subtle, as in the two patients of our cohort; thus, it needs to be actively investigated, especially in high-risk patients. An early recognition of inflammatory myocardial involvement is indeed of cardinal importance to allow a prompt therapeutic intervention, thus improving patient's outcome.

Given the concomitant presence of elevated CK in all anti-Ku patients in our cohort, we suggest that not only ILD but also myocarditis, might be a specific feature of the anti-Ku with elevated CK subgroup and that all anti-Ku-positive SSc patients should be actively screened for potential myocardial involvement.

\section{Corrado Campochiaro, ${ }^{1}$ Giacomo De Luca, ${ }^{1}$ Maria De Santis ${ }^{2}$}

${ }^{1}$ Unit of Immunology, Rheumatology, Allergy and Rare Diseases IRCCS San Raffaele Hospital. Università Vita-Salute San Raffaele, San Raffaele Scientific Institute, Milan, Italy

${ }^{2}$ Division of Rheumatology and Clinical Immunology, Humanitas Clinical and Research Center - IRCCS, Milan, Italy

Correspondence to Dr Corrado Campochiaro, Università Vita-Salute San Raffaele, School of Medicine; Unit of Immunology, Rheumatology, Allergy and Rare Diseases, San Raffaele Scientific Institute, Milan 20132, Italy; campochiaro.corrado@hsr.it

Contributors CC: conceived the hypothesis, analysed data and drafted the manuscript. GDL: conceived the hypothesis, analysed data, critically revised the manuscript and gave the final approval. MDS: analysed data, critically revised the manuscript and gave the final approval.

Funding The authors have not declared a specific grant for this research from any funding agency in the public, commercial or not-for-profit sectors.

Competing interests None declared.

Patient consent for publication Not required.

Provenance and peer review Not commissioned; internally peer reviewed.

(c) Author(s) (or their employer(s)) 2019. No commercial re-use. See rights and permissions. Published by BMJ.

\section{Check for updates}

To cite Campochiaro C, De Luca G, De Santis M. Ann Rheum Dis Epub ahead of print: [please include Day Month Year]. doi:10.1136/annrheumdis-2019-216070

Received 23 July 2019

Accepted 27 July 2019

Ann Rheum Dis 2019;0:1. doi:10.1136/annrheumdis-2019-216070

\section{REFERENCES}

1 Spielmann L, Nespola B, Séverac F, et al. Anti-Ku syndrome with elevated CK and antiKu syndrome with anti-dsDNA are two distinct entities with different outcomes. Ann Rheum Dis 2019;78:1101-6.

2 van den Hoogen F, Khanna D, Fransen J, et al. 2013 classification criteria for systemic sclerosis: an American College of rheumatology/European League against rheumatism collaborative initiative. Ann Rheum Dis 2013;72:1747-55.

3 Friedrich MG, Sechtem U, Schulz-Menger J, et al. Cardiovascular magnetic resonance in myocarditis: a JACC white paper. J Am Coll Cardiol 2009;53:1475-87.

4 Follansbee WP, Zerbe TR, Medsger TA. Cardiac and skeletal muscle disease in systemic sclerosis (scleroderma): a high risk association. Am Heart J 1993;125:194-203.

5 Pieroni M, De Santis M, Zizzo G, et al. Recognizing and treating myocarditis in recentonset systemic sclerosis heart disease: potential utility of immunosuppressive therapy in cardiac damage progression. Semin Arthritis Rheum 2014;43:526-35. 